\title{
The role of EGFR-targeting strategies in the treatment of head and neck cancer
}

This article was published in the following Dove Press journal:

OncoTargets and Therapy

26 July 2012

Number of times this article has been viewed

\section{Didier Dequanter \\ Mohammad Shahla \\ Pascal Paulus \\ Philippe H Lothaire}

Department of Surgery, $\mathrm{CHU}$ Charleroi (Hopital Andre Vésale), Montigny le Tilleul, Belgium
Correspondence: Didier Dequanter Maandal 6, 1652 Alsemberg, Belgium Tel +32 07। 92I 5II

Email didier.dequanter@pandora.be
Abstract: With its targeted mechanism of action and synergistic activity with current treatment modalities, cetuximab is a potentially valuable treatment option for patients with recurrent and/or metastatic squamous cell cancer of the head and neck who have progressed on cisplatin-based chemotherapy. The use of cetuximab in combination with radiotherapy as definitive treatment for locoregionally advanced squamous cell cancer of the head and neck is generally restricted to patients unfit to receive cisplatin-based chemoradiation, which is still considered the standard of care. The effect of this epidermal growth factor receptor antagonist occurs without any change in the pattern and the severity of toxicity usually associated with head and neck radiation.

Keywords: cetuximab, SCCHN, radiotherapy

\section{Introduction}

As $90 \%$ of all cases of squamous cell cancer of the head and neck show expression of epidermal growth factor receptor (EGFR), there is a strong rationale supporting EGFR-targeting in this malignancy. ${ }^{1,2}$ The EGFR molecule consists of an N-terminal, extracellular, ligand-binding domain; a hydrophobic, transmembrane domain, and; a C-terminal, intracellular domain with tyrosine kinase activity. EGFR is a $170-\mathrm{kD}$ transmembrane glycoprotein encoded by the c-ErbB1 proto-oncogene, which is situated on the 7q22 chromosome. The EGFR can be stimulated by several growth factors such as transformation growth factor alpha and epidermal growth factor, which bind to the extracellular domain of the receptor. Other known ligands of EGFR include amphiregulin, heparin-binding EGRF, betacellulin, epiregulin, and neuregulin-2-alpha. This ligand binding results in EGFR dimerization and activation of the tyrosine kinase domains present on each receptor. In turn, kinase activation results in cross-phosphorylation of tyrosine residues on each member of the receptor repair, and initiates the formation of signaling complexes in the cytoplasm, which can translocate to the cell nucleus and activate gene transcription; this editing of gene transcription eventually results in the induction of several cellular responses including, cell proliferation. Ultimately, receptor ligand complexes are internalized and the signal is terminated. ${ }^{3}$

In preclinical models, it was found that inhibition of EGFR had antitumor activity; available data suggested treatment synergy between EGFR-targeted chemotherapy and radiotherapy. ${ }^{4}$ In current clinical practice, there are two EGFR-targeting strategies employing monoclonal antibodies directed at the extracellular domain of the receptor, and small molecule, ATP tyrosine kinase inhibitors. ${ }^{5,6}$ The use of both modalities 
in the treatment of squamous cell cancer of the head and neck (SCCHN) is currently under investigation.,

\section{Cetuximab}

Thus far, the monoclonal EGFR antibody cetuximab has been the most studied in detail. Cetuximab is a human-murine chimeric immunoglobulin G1 monoclonal antibody, which competitively binds to the extracellular domain of the EGFR, preventing activation of the receptor by endogenous ligands. The antibody-receptor complex is internalized and degraded, resulting in a downregulation of the EGFR expression.

Cetuximab has been tested in first-line treatment, in combination with radiation, as definitive treatment for locoregionally advanced SCCHN. Hence, the rationale for combination radiotherapy with cetuximab in SCCHN has been demonstrated. ${ }^{9}$

In an initial study, cetuximab showed encouraging activity in patients with locally advanced SCCHN. In this Phase I trial, ${ }^{10} 16$ patients with advanced SCCHN received treatment with cetuximab combined with either conventional or hyperfractionated radiotherapy. There was an impressive $100 \%$ treatment-response rate (13 complete and three partial responses) and interestingly, both treatments were generally well tolerated (Table 1).

Results showed better survival and locoregional disease control associated with cetuximab plus radiotherapy than with radiotherapy alone; further, these differences were not associated with reduced quality of life, nor increased radiation-induced mucositis and dysphagia.

In another, multinational, randomized study, ${ }^{11} 424$ patients with locoregionally advanced SCCHN were randomized to receive ether radiotherapy alone, or radiotherapy combined with weekly cetuximab. Patients with stage 3 or 4 , nonmetastatic, measurable squamous cell carcinoma of the oropharynx, hypopharynx, or larynx were eligible. The primary endpoint was the duration of control of locoregional disease. Secondary endpoints were overall survival, progression-free survival, the response rate, and safety. At the end of study, the median duration of locoregional control was 24.4 months in the group receiving combined therapy compared with 14.9 months in the group receiving radiotherapy alone $(P=0.005)$. The $1-, 2-$, and 3-year rates of locoregional control achieved with radiotherapy plus cetuximab $(63 \%, 50 \%$, and $47 \%$, respectively) were significantly higher than those achieved with radiotherapy alone $(55 \%, 41 \%$, and $34 \%$, respectively) $(P<0.01)$. After a median follow-up period of 54 months, the median survival time was 49 months among patients treated with combined therapy versus 29.3 months among those treated with radiotherapy alone $(P=0.03)$.

A second, later analysis was undertaken to assess 5-year overall survival results. ${ }^{12}$ The longer-term results corroborated the earlier findings that the addition of cetuximab to radiotherapy improved the survival of patients with SCCHN. The addition of cetuximab to radiotherapy resulted in an increase of $9.2 \%$ in absolute survival (45.6\% versus $36.4 \%$ ), compared with radiotherapy alone. Further subgroup analysis showed that patients given cetuximab who developed prominent cetuximab-induced acneiform rash (grades 2-4) had better overall survival than those who developed a mild or no rash (grades $0-1$ ). In this second study, ${ }^{12}$ the overall survival benefit associated with the addition of cetuximab to radiotherapy relative to radiotherapy alone, was remarkably similar to that seen in the 3 -year analysis (45\% versus $55 \%$ ) (which supports the validity of 3 -year survival as a prognosticator of long-term overall survival).

Cetuximab has also been tested in metastatic and/or recurrent SCCHN as a second line of treatment, after failure of platinum-based chemotherapy. ${ }^{7,8}$ Three Phase II trials examined the role of cetuximab in platinum-refractory or resistant disease. All patients received cetuximab intravenously at an initial loading dose of $400 \mathrm{mg} / \mathrm{m}^{2}$, followed by a weekly dose of $250 \mathrm{mg} / \mathrm{m}^{2}$.

Baselga et $\mathrm{al}^{13}$ added weekly cetuximab to platinumbased chemotherapy in 96 patients with SCCHN who progressed after a minimum of two, and a maximum of four cycles of cisplatin- or carboplatin-based chemotherapy.

Table I Activity of cetuximab in combination with radiation and platinum in refractory recurrent and/or metastatic SCCHN

\begin{tabular}{|c|c|c|c|c|}
\hline Authors & Treatment & No of patients & Response rate (\%) & Median survival (months) \\
\hline \multicolumn{5}{|c|}{ Activity of cetuximab in combination with radiation } \\
\hline Robert et al ${ }^{10}$ & Cetuximab + radiotherapy & 16 & 100 & - \\
\hline Bonner et al ${ }^{12}$ & Cetuximab \pm radiotherapy & 424 & - & 49 \\
\hline \multicolumn{5}{|c|}{ Cetuximab in combination with platinum-based chemotherapy } \\
\hline Baselga et $\mathrm{a}^{13}$ & Cetuximab + cisplatin/carboplatin monotherapy & 96 & 10 & 6.1 \\
\hline Vermorken et al ${ }^{15}$ & Cetuximab + cisplatin & 103 & 13 & 4.1 \\
\hline Herbst et al ${ }^{14}$ & Cetuximab + cisplatin & 130 & 13 & 7.4 \\
\hline
\end{tabular}

Abbreviation: SCCHN, squamous cell carcinoma of the head and neck. 
Results confirmed that all of the patients had truly platinumrefractory disease. The objective response rate was $10 \%$, and all responses were incomplete. The disease control rate was $53 \%$. The median time to progression and overall survival rate were 85 and 183 days, respectively.

Vermorken et $\mathrm{al}^{14}$ conducted an open-label, uncontrolled, multicenter, Phase II study with a two-phase design. In the first phase, 103 patients with metastatic or recurrent SCCHN, who progressed within the 30 days following a minimum of two cycles of platinum-based chemotherapy, received singleagent cetuximab. If possible, treatment was continued for at least 6 weeks and was sustained until progression of disease, clinical deterioration, or unacceptable adverse events were observed. A partial response was observed in $13 \%$ of the patients. The disease control was rate was $46 \%$. The median duration of response and the median time to progression were 126 and 70 days, respectively. In the second phase of the trial, 53 patients $(51 \%)$ who progressed while receiving single-agent cetuximab, continued treatment with cetuximab in combination with platinum compound. In this second phase, no objective responses were observed. The median time to progression was 50 days. The median overall survival rate was 178 days.

Herbst et $\mathrm{al}^{15}$ studied the combination of cetuximab and chemotherapy in a more heterogeneous population of 130 patients with recurrent and/or metastatic SCCHN. The patients had either stable disease after 2 cycles, or had progression under cisplatin-based chemotherapy. After cetuximab was added to the existing regimen, $13 \%$ of the patients responded; again, all responses were incomplete. The disease control rate in the patients with progressive disease was 55\%. Median duration of response was approximately 4 months in patients with progressive disease, and 7.4 months in patients with stable disease.

Cetuximab has also been tested in metastatic and/or recurrent SCCHN, as a first-line treatment, in combination with platinum-based therapy. The feasibility of combining cetuximab with cisplatin, or carboplatin and 5-fluorouracil, was demonstrated in a Phase III study. ${ }^{16}$ In that study, the overall response rate was $36 \%$. The potential benefit was also studied in two randomized multicenter Phase III trials. ${ }^{17-19}$

In the EXTREME study, 442 patients were randomized to receive $100 \mathrm{mg} / \mathrm{m}^{2}$ cisplatin or carboplatin at an AUC 5 on day 1 , followed by 5 -fluorouracil at $1000 \mathrm{mg} / \mathrm{m}^{2} /$ day for 4 days, alone or combined with weekly cetuximab. ${ }^{18}$ Cycles were repeated every 3 weeks for a maximum of six cycles. In the combination arm, cetuximab was continued until disease progression or unacceptable toxicity was observed.
Patients with recurrent and/or metastatic SCCHN, with measurable disease and a Karnofsky score of at least 70 were eligible. The addition of cetuximab increased the median survival by 2.7 months from 7.4 to 10.1 months (hazard ratio $=0.797 ; P=0.036$ ). With the addition of cetuximab, the response rate increased from $19.5 \%$ to $35.6 \%(P<0.0001)$, and the median progression-free survival was prolonged by 2.3 months, from 3.3 to 5.6 months $(P<0.0001)$. The addition of cetuximab did not affect the characteristic adverse event profile of platinum-based chemotherapy.

Burtness et al assigned 117 patients to a regimen of cisplatin, $100 \mathrm{mg} / \mathrm{m}^{2}$ every four weeks in combination with weekly cetuximab or placebo. ${ }^{17}$ Patients were stratified for performance status and disease status (previously treated or untreated); $30 \%$ of the patients in the cisplatin plus cetuximab arm, versus $15 \%$ in the cisplatin plus placebo arm, had received prior systemic therapy, either as adjuvant or induction chemotherapy. The primary endpoint was progression-free survival (PFS). The study was designed to detect a difference in median PFS from 2 months with cisplatin plus placebo to 4 months with the experimental arm with $90 \%$ power using a one-sided long-rank test with an overall type I error rate of 0.025 . The observed PFS in the control arm was 2.7 months, versus 4.2 months in the cetuximab arm, and this difference did not reach statistical significance $(P=0.09)$. However, due to longer survival in the control arm, the power to detect a 2-month difference in PFS was decreased to $50 \%$. The objective response rate was $26 \%$ for the experimental arm versus $10 \%$ in the control arm $(P=0.03)$. Median overall survival was not significantly different in the two groups $(9.2$ months versus 8 months, $P=0.21)$. Notably, development of cetuximab-related skin toxicity was associated with an improved overall survival (hazard ratio $0.42, P=0.01$ ).

Vermorken et a ${ }^{19}$ randomly assigned 220 of 442 eligible patients with untreated, recurrent, or metastatic squamous cell carcinoma of the head and neck to receive cisplatin or carboplatin plus fluorouracil every 3 weeks for a maximum of six cycles, and 222 patients to receive the same chemotherapy plus cetuximab for a maximum of six cycles. Patients in the cetuximab arm continued to receive cetuximab until disease progression or unacceptable toxic effects, whichever occurred first. Adding cetuximab to platinum-based chemotherapy with fluorouracil significantly prolonged the median overall survival from 7.4 months in the chemotherapyalone group, to 10.1 months in the group that received chemotherapy plus cetuximab (hazard ratio $=0.80,95 \%$ confidence interval: $0.64,0.99 ; P=0.04)$. The addition of 
cetuximab prolonged the median progression-free survival time from 3.3 months to 5.6 months (hazard ratio $=0.54$, $P<0.001)$, and increased the response rate from $20 \%$ to $36 \%(P<0.001)$.

The development of acneiform rash is a toxicity that is frequently associated with cetuximab and not with radiotherapy alone. The characteristic cetuximab-induced acneiform rash arises during treatment and generally resolves completely in the first weeks following the cessation of treatment. ${ }^{20}$ In a study of 208 patients who received cetuximab, 174 (84\%) had a rash and 127 patients had a prominent rash. This incidence was similar to other previous reports of treatment with cetuximab alone. ${ }^{21}$ Patients with a prominent rash had a greater than 2.5 times longer overall survival than did patients with a mild rash.

Notably, cetuximab has not been shown to dramatically exacerbate the common toxic effects associated with radiotherapy of the head and neck, including mucositis, xerostomia, dysphagia, pain, weight loss, and performance status deterioration; however, a few cases of severe skin reactions within the radiation field have been reported. ${ }^{19}$ As in other tumors, the occurrence of grade $2-4$ skin toxicity in SCCHN patients treated with cetuximab has been associated with better outcome. ${ }^{15,17}$

Cetuximab appears to provide the greatest benefit for patients with oropharyngeal tumors, T1-3 tumors, concomitant boost, advanced nodal stage, high Karnofsky Performance scores, and for patients receiving treatment in the USA. ${ }^{12}$

\section{Other EGFR-directed agents}

The use of several other EGFR-directed monoclonal antibodies and EGFR tyrosine kinase inhibitors is currently under investigation for the treatment of SCCHN. ${ }^{3,5}$ The best studied, are gefitinib and erlotinib. In the recurrent/metastatic disease setting the response rate with these agents ranges between $3 \%$ and $10 \%$. A higher response rate has been observed with gefinitib (500 mg/day) compared with gefinitib (250 mg/day); however, this difference has not translated to a better overall rate of survival (Table 2).

To date, erlotinib has been safely combined with chemoradiation without requiring a dose reduction of either chemo- or radiotherapy. In a Phase I study, patients received increasing doses of erlotinib (100-150 mg/day orally) and cisplatin (30-40 mg/m²/day intravenously) for 7 weeks, in combination with a standard radiotherapy regimen (1.8 Gy daily [five fractions/week] to a total dose of 63 Gy in 7 weeks), and the regimen was well tolerated. ${ }^{22}$
Table 2 EGFR-targeting agents under clinical investigation in head and neck cancer

\begin{tabular}{|c|c|c|c|c|}
\hline & & & & Toxicity \\
\hline \multicolumn{5}{|c|}{ Monoclonal antibodies } \\
\hline Cetuximab & IMC225 & $\lg G \mid$ & $\begin{array}{l}\text { Chimeric } \\
\text { human/murine }\end{array}$ & Skin \\
\hline Matuzumab & EMD72000 & $\operatorname{lgGI}$ & $\begin{array}{l}\text { Humanized } \\
\text { mouse }\end{array}$ & Skin \\
\hline Nimotuzumab & h-R3 & $\lg G \mid$ & $\begin{array}{l}\text { Humanized } \\
\text { mouse }\end{array}$ & Systemic \\
\hline Zalutumumab & $2 \mathrm{~F} 8$ & $\lg G \mid$ & Human & Skin \\
\hline Panitumumab & ABX-EGF & $\lg G 2$ & Human & Skin \\
\hline \multicolumn{5}{|c|}{ Tyrosine kinase inhibitors } \\
\hline Gefinitib & ZDI839 & EGFR & Reversible & $\begin{array}{l}\text { Skin/ } \\
\text { gastrointestinal }\end{array}$ \\
\hline Erlotinib & OSI-774 & EGFR & Reversible & $\begin{array}{l}\text { Skin/ } \\
\text { gastrointestinal }\end{array}$ \\
\hline Lapatinib & GW-572016 & $\begin{array}{l}\text { EGFR/ } \\
\text { ERbB2 }\end{array}$ & Reversible & Skin/hepatic \\
\hline Canertinib & $\mathrm{Cl}-0033$ & EGFR & Irreversible & $\begin{array}{l}\text { Skin/oral/ } \\
\text { systemic }\end{array}$ \\
\hline
\end{tabular}

Abbreviation: EGFR, epidermal growth factor receptor.

A Phase II trial conducted by the Sarah Cannon Oncology Research Consortium investigated combined modality treatment with erlotinib, bevacizumab, chemotherapy, and radiotherapy in patients with locally advanced SCCHN. The addition of bevacizumab and erlotinib to first-line combinedmodality therapy, proved to be feasible, producing toxicity that was comparable to other effective combined-modality regimens for head and neck cancer. ${ }^{23}$

It is likely that in future, renewed interest in induction chemotherapy will stimulate investigation of the feasibility and efficacy of combining induction chemotherapy with EGFR-directed therapies. ${ }^{24,25}$

\section{Conclusions}

The use of cetuximab in combination with radiotherapy as definitive treatment for locoregionally advanced SCCHN is generally restricted to patients unfit to receive cisplatin-based chemoradiation, which is still considered to be the standard of care. However, cetuximab has been shown to synergize with current treatment modalities and as such, is a potentially valuable treatment option for patients with recurrent and/or metastatic SCCHN, who have progressed on cisplatin-based chemotherapy. Moreover, the effect of the epidermal growth factor receptor (EGFR) antagonist occurs without any change in the pattern, or the severity, of toxicity usually associated with head and neck radiation.

\section{Disclosure}

The authors report no conflict of interest in this work. 


\section{References}

1. Saleh MN, Raisch KP, Stackhouse MA, et al. Combined modality therapy of A431 human epidermoid cancer using anti-EGFR antibody C225 and radiation. Cancer Biother Radiopharm. 1999;14(6):451-463.

2. Rapidis AD, Vermorken JB, Bourhis J. Targeted therapies in head and neck cancer: past, present and future. Rev Recent Clin Trials. 2008;3(3): $156-166$

3. Specenier P, Vermorken JB. Targeted therapies in head and neck cancer. Target Oncol. 2007;2:73-88.

4. Grandis JR, Tweardy DJ. Elevated levels of transforming growth factor alpha and epidermal growth factor receptor messenger RNA are early markers of carcinogenesis in head and neck cancer. Cancer Res. 1993;53(15):3579-3584.

5. Specenier PM, Vermorken JB. Recurrent head and neck cancer: current treatment and future prospects. Expert Rev Anticancer Ther. 2008;8(3):375-391.

6. Cruz JJ, Ocaña A, Del Barco E, Pandiella A. Targeting receptor tyrosine kinases and their signal transduction routes in head and neck cancer. Ann Oncol. 2007;18(3):421-430.

7. Brockstein B, Lacouture M, Agulnik M. The role of inhibitors of the epidermal growth factor in management of head and neck cancer. J Natl Compr Canc Netw. 2008;6(7):696-706.

8. Langer CJ. Targeted therapy in head and neck cancer: state of the art 2007 and review of clinical applications. Cancer. 2008;112(12): 2635-2645.

9. Bernier J, Schneider D. Cetuximab combined with radiotherapy: an alternative to chemoradiotherapy for patients with locally advanced squamous cell carcinomas of the head and neck? Eur J Cancer. 2006; 43(1):35-45.

10. Robert F, Ezekiel MP, Spencer SA, et al. Phase I study of anti epidermal growth factor receptor antibody cetuximab in combination with radiation therapy in patients with advanced head and neck cancer. J Clin Oncol. 2001;19(13):3234-3243.

11. Bourhis J, Rivera F, Mesia R, et al. Phase I/II study of cetuximab in combination with cisplatin or carboplatin and fluorouracil in patients with recurrent or metastatic squamous cell carcinoma of the head and neck. J Clin Oncol. 2006;24(18):2866-2872.

12. Bonner JA, Harari PM, Giralt J, et al. Radiotherapy plus cetuximab for locoregionally advanced head and neck cancer: 5-year survival data from a phase 3 randomised trial, and relation between cetuximabinduced rash and survival. Lancet Oncol. 2010;11(1):21-28.

13. Baselga J, Trigo JM, Bourhis J, et al. Phase II multicenter study of the antiepidermal growth factor receptor monoclonal antibody cetuximab in combination with platinum-based chemotherapy in patients with platinum-refractory metastatic and/or recurrent squamous cell carcinoma of the head and neck. J Clin Oncol. 2005;23(24):5568-5577.

14. Vermorken JB, Trigo J, Hitt R, et al. Open-label, uncontrolled, muliticenter phase II study to evaluate the efficacy and toxicity of cetuximab as a single agent in patients with recurrent and/or metastatic squamous cell carcinoma of the head and neck who failed to respond to platinumbased therapy. J Clin Oncol. 2007;25(16):2171-2177.
15. Herbst RS, Arquette M, Shin DM, et al. Phase II multicenter study of the epidermal growth factor receptor antibody cetuximab and cisplatin for recurrent and refractory squamous cell carcinoma of the head and neck. J Clin Oncol. 2005;23(24):5578-5587.

16. Bonner J, Harari PM, Giralt J. The relationship of cetuximab-induced rash and survival in patients with head and neck cancer treated with radiotherapy and cetuximab. Int J Radiat Oncol Biol Phys. 2005;63:S73.

17. Burtness B, Goldwasser MA, Flood W, Mattar B, Forastiere AA; for Eastern Cooperative Oncology Group. Phase III randomized trial of cisplatin plus placebo compared with cisplatin plus cetuximab in metastatic/recurrent head and neck cancer: an Eastern Cooperative Oncology Group Study. J Clin Oncol. 2005;23(34):8646-8654.

18. Vermorken JB, Mesia R, Vega-Villlegas ME, Remenar E, Hitt R, Kawecki A. Cetuximab extends survival of patients with recurrent and/or metastatic squamous cell carcinoma of the head and neck when added to first line platinum based therapy. Results of a randomized phase III trial (EXTREME) KJ [abstract]. Clin Oncol. 2007; 25(18S):298s; 6091.

19. Vermorken JB, Mesia R, Rivera F, et al. Platinum-based chemotherapy plus cetuximab in head and neck cancer. $N$ Engl J Med. 2008;359(11): $1116-1127$.

20. Bonner JA, Harrari PM, Giralt J, et al. Radiotherapy plus cetuximab for squamous cell carcinoma of the head and neck. $N$ Eng $J$ Med. 2006;354(6):567-578.

21. Lenz HJ, Van Cutsem E, Khambata-Ford S, et al. Multicenter phase II and translational study of cetuximab in metastatic colorectal carcinoma refractory to irinotecan, oxaliplatin, and fluoropyrimidines. J Clin Oncol. 2006;24(30):4914-4921.

22. Budach W, Bölke E, Homey B. Severe cutaneous reaction during radiation therapy with concurrent cetuximab. $N$ Eng J Med. 2007;357(5): 514-515.

23. Arias de la Vega F, Contreras J, de Las Heras M, et al; for Members of GICOR (Grupo de Investigación Clínica en Oncología Radioterápica) group. Erlotinib and chemoradiation in patients with surgically resected locally advanced squamous cell carcinoma of the head and neck: a GICOR phase I trial. Ann Oncol. 2012;23(4):1005-1009.

24. Hainsworth JD, Spigel DR, Greco FA, et al. Combined modality treatment with chemotherapy, radiation therapy, bevacizumab, and erlotinib in patients with locally advanced squamous carcinoma of the head and neck: a phase II trial of the Sarah Cannon oncology research consortium. Cancer J. 2011;17(5):267-272.

25. Vermorken JB, Remenar E, van Herpen C, et al; for EORTC 24971/TAX 323 Study Group. Cisplatin, fluorouracil, and docetaxel in unresectable head and neck cancer. N Engl J Med. 2007;357(17):1695-1704.

26. Posner MR, Hershock DM, Blajman CR, et al; for TAX 324 Study Group. Cisplatin and fluorouracil alone or with docetaxel in head and neck cancer. $N$ Engl J Med. 2007;357(17):1705-1715.
OncoTargets and Therapy

\section{Publish your work in this journal}

OncoTargets and Therapy is an international, peer-reviewed, open access journal focusing on the pathological basis of all cancers, potential targets for therapy and treatment protocols employed to improve the management of cancer patients. The journal also focuses on the impact of management programs and new therapeutic agents and protocols on

Submit your manuscript here: http://www.dovepress.com/oncotargets-and-therapy-journal

\section{Dovepress}

patient perspectives such as quality of life, adherence and satisfaction. The manuscript management system is completely online and includes a very quick and fair peer-review system, which is all easy to use. Visit http://www.dovepress.com/testimonials.php to read real quotes from published authors. 